\title{
Association between G-protein coupled receptor 4 expression and microvessel density, clinicopathological characteristics and survival in hepatocellular carcinoma
}

\author{
CHAOFAN XUE ${ }^{1}$, SHUAI SHAO ${ }^{1}$, YANLI YAN ${ }^{1}$, SI YANG $^{1}$, SHUHENG BAI $^{1}$, YINYING WU ${ }^{2}$, \\ JIANGZHOU ZHANG ${ }^{1}$, RUI LIU ${ }^{1}$, HAILIN MA ${ }^{1}$, LINYAN CHAI ${ }^{1}$, XIAOZHI ZHANG ${ }^{1}$ and JUAN REN $^{1}$ \\ Departments of ${ }^{1}$ Radiotherapy and ${ }^{2}$ Chemotherapy, Oncology Department, \\ First Affiliated Hospital of Xi'an Jiaotong University, Xi'an, Shaanxi 710061, P.R. China
}

Received November 2, 2018; Accepted June 14, 2019

DOI: $10.3892 / \mathrm{ol} .2020 .11366$

\begin{abstract}
G-protein coupled receptor 4 (GPR4) acts as a proton-sensing receptor and plays a role in regulating angiogenesis. Endoglin/CD105 is a marker of cell proliferation in vascular endothelial cells, particularly in tumor vasculature cells. Although there have been several studies investigating angiogenesis in hepatocellular carcinoma (HCC), none have investigated the association between GPR4 and microvessel density (MVD)-CD105 in this type of cancer. In the present study, CD105 and GPR4 were found to be expressed in benign and malignant liver tissues by immunofluorescence staining and laser confocal microscopy. Compared with levels in benign tissues, CD105 and GPR4 were highly expressed in neoplastic tissues. Furthermore, the average fluorescence intensity of GPR4 and MVD-CD105 was positively correlated. GPR4 and CD105 were found to be co-localized in the vascular endothelium in tumor tissues. Furthermore, the expression of GPR4 was higher in the marginal region of tumor tissues compared with the central region. These findings suggest that the expression of GPR4 in tumor microvessels in HCC may be implicated in tumor angiogenesis and development. Furthermore, the association between the expression of GPR4 and the clinicopathological features of patients with $\mathrm{HCC}$ further suggests a role for GPR4 in tumor angiogenesis and growth. Overall, these results suggest the potential of GPR4 as a prognostic factor and as an antiangiogenic target in patients with HCC.
\end{abstract}

Correspondence to: Dr Juan Ren, Department of Radiotherapy, Oncology Department, First Affiliated Hospital of Xi'an Jiaotong University, 277 Yanta West Road, Xi'an, Shaanxi 710061, P.R. China E-mail: 869491533@qq.com

Key words: G-protein coupled receptor 4, CD105, microvessel density, angiogenesis, hepatocellular carcinoma

\section{Introduction}

The G-protein-coupled receptor (GPCR) family is the largest family in the human genome and the most widely distributed membrane protein receptor in humans. The ovarian cancer GPCR 1 (OGR1) subfamily is a family of proton-sensing receptor proteins. The four members of the proton-activated GPCR family, GPCR 1 (OGR1), GPCR 4 (GPR4), T-cell death-associated gene 8 and GPCR 2A have been also identified as receptors of some specific lipid molecules, including sphingosine phosphoryl choline and lysophosphatidyl choline (1-4).

GPR4 is overexpressed in lung tissues, with lower expression in the kidney, heart, skeletal muscle and pancreatic tissues (5). GPR4 was firstly described as a proton-sensing receptor in 2003 (1); it was found to be involved in a variety of biological processes (1), including the inhibition of cell apoptosis, the promotion of cell proliferation and metastasis by initiating different signaling pathways, and the induction of cytosolic cyclic adenosine monophosphate (cAMP) in cells by recognizing and accepting protons via its unique extracellular histidine residues (6). Additionally, GPR4 plays an important role in angiogenesis, and the proangiogenic effect is independent of its ligand-hemolytic phospholipids. In previous studies, it was found that GPR4 induced proliferation and migration of vascular endothelial cells (ECs), as well as angiogenesis (7). Following GPR4-knockout, vascular ECs had lower survival, decreased migration and were unable to form tubular structures. These effects were reversed when GPR4 gene was re-expressed (8). These findings suggest that GPR4 plays an important role in angiogenesis. Furthermore, certain studies have demonstrated the ability of GPR4 to induce angiogenesis in ovarian cancer and squamous cell carcinoma of the head and neck (SCCHN) $(9,10)$. In ovarian cancer, the expression level of GPR4 is positively correlated with the microvessel density (MVD) of vascular ECs, which is not observed in benign ovarian tissues (10). In SCCHN, GPR4 promotes angiogenesis by inducing $\mathrm{p} 38$, which mediates secretion of vascular endothelial growth factor A, interleukin (IL)-6 and IL-8 (9). In mice with conditional loss of GPR4 expression, the expression level of vascular endothelial growth factor 
(VEGF) receptor 2 was decreased, with suppressed angiogenesis that remained low following re-expression of VEGF (11). Furthermore, GPR4-deficient embryos and neonates had a high perinatal mortality rate (12). Moreover, some GPR4-null embryos and neonates presented with a tendency for spontaneous bleeding. In addition, a proportion of subcutaneous blood vessels became dilated and tortuous with defective vascular smooth muscle cell coverage, potentially resulting in impaired vascular stability and abnormal bleeding (12).

Sin et al (13) found that GPR4 was an oncogene that was overexpressed in human cancer tissues of the breast, ovary, colon, liver and kidney. Mouse NIH3T3 fibroblasts transfected with GPR4, resulted in increased survival at low serum concentrations (13). Furthermore, the neoplastic transformation of NIH3T3 was found to be successfully induced through GPR4 transfection (13). Additionally, a study in a nude mouse model also demonstrated the carcinogenic ability of GPR4 (11). Wyder et al (11) reported that breast cancer and colon carcinoma growth were significantly inhibited in GPR4-deficient mice. Moreover, cell proliferation, invasion and metastatic ability were significantly attenuated in ovarian cancer following GPR4-knockout, suggesting that this gene plays a role in regulating proliferation, metastasis and invasion of ovarian cancer.

Hepatocellular carcinoma (HCC) is a malignant solid tumor enriched with blood vessels, with a unique vascular distribution. HCC tumors are mainly supplied by the hepatic artery, and the blood supply in the normal liver parenchyma is mainly derived from the portal vein and the hepatic artery $(14,15)$. Tumors of the liver grow fast and are associated with a poor prognosis due to the abundant blood supply. Thus, angiogenesis is an important therapeutic target in HCC. Hematogenous and distant metastases are the major types of metastases in HCC. Under the microscope, the blood vessels of HCC appear abnormal, mainly arterial and with sinusoidal capillaries compared with normal blood vessels (16). Due to the high metabolism associated with HCC, the tumor tissue is mainly fed by the arterial system. Overgrowth of the artery can be part of the non-invasive diagnostic criteria for HCC (17). Hepatic sinusoidal capillarization is another angiogenic feature that is commonly found in HCC. Additionally, the liver sinusoidal ECs are characterized by decreased hepatic sinusoidal fenestration and continuous capillary formation, along with collagenization of the sinusoids, laminin deposition near ECs and hepatocytes, and formation of the basement membrane (18). Certain studies have suggested a role for GPCRs in tumorigenesis and the development of HCC (19). Aberrant expression of GPCRs, such as C-X-C chemokine receptor type 7, promotes HCC tumor growth by regulating signaling pathways that promote cell migration, metastases formation and angiogenesis (20). At present, to the best of our knowledge, there are no studies that have investigated the association between GPR4 expression and tumor MVD in HCC.

As a member of the superfamily of transforming growth factor $\beta$ receptors (21), endoglin/CD105 is a marker of cell proliferation in vascular ECs, particularly in the tumor vasculature (21-24). In human breast cancer, CD105 and CD34 antibodies have been used to assess MVD. MVD, quantified by CD105, demonstrated an association between the overall survival time (OS) of the patient and the expression of CD34 (22). The MVD of tumor tissues not only reflects tumor blood vessel growth quantitatively, but also acts as an independent prognostic factor in some solid tumors (25).

In the present study, CD105 was used to mark blood vessels and to determine the MVD. Furthermore, the expression of GPR4 and its co-localization with CD105 was determined in HCC tumors. The association between expression levels of GPR4 in HCC cells and the clinicopathological characteristics of patients with $\mathrm{HCC}$ was also investigated.

\section{Materials and methods}

Clinical data collection. A total of 47 specimens were obtained from The First Affiliated Hospital of Xi'an Jiaotong University (Xi'an, Shaanxi, China) between November 2014 and June 2016. The specimens included 37 cases of HCC and 10 cases of non-HCC (including 6 hepatic hemangioma samples and 4 normal liver samples obtained 2-5 cm away from the liver tumor tissue) as controls. In each patient, both tumor and adjacent normal liver tissue were obtained. The definition of adjacent normal tissue is generally considered to be $2-5 \mathrm{~cm}$ away from the tumor. However, in clinical practice, the criteria for the diagnosis and radical resection of primary $\mathrm{HCC}$ indicate that the distance between the liver resection margin and the tumor boundary should be $>1 \mathrm{~cm}$. Thus, the ideal distance for paracancerous tissue was noted in a smaller sample number than expected. Therefore, liver tissues $>2 \mathrm{~cm}$ away from the liver tumor and normal liver tissues obtained from the resection of the hepatic hemangioma were included in the control group (26). All clinical data of patients with liver cancer were collected and thoroughly analyzed. Tumor staging before chemotherapy and radiotherapy was performed according to the 2010 United States Cancer Board (American Joint Committee on Cancer) system standard (27). Clinicopathological data, including sex, age, surgical duration, tumor size, lymph node metastases and pathological type, were retrieved from the electronic medical records. The 37 patients were aged between 28 and 81 years, with an average of $55.59 \pm 11.14$ years. A total of 8 cases were well-differentiated carcinomas, 24 cases were moderately differentiated carcinomas and 5 cases were poorly differentiated carcinomas. The study was approved by the Ethics Committee of the First Affiliated Hospital of Xi'an Jiaotong University. The median follow-up time was 14.5 months and the study endpoint was determined by the patient's death. OS time was used as a prognostic indicator.

Hematoxylin-eosin staining of frozen sections. Tissue sections were permeabilized with PBS-Tween-20 (Beijing Dingguo Changsheng Biotechnology Co., Ltd.) and double distilled water. Hematoxylin nuclei were stained at room temperature for 3-5 min, and the nuclei were observed to turn blue under the microscope. After that, the hematoxylin dyes were removed with double steaming water, followed by $1 \%$ hydrochloric acid - alcohol separation for $3-5 \mathrm{sec}$. The slices turned blue following the addition tap water. The slides were stained with eosin for 1-2 min at room temperature, and the slides were dehydrated and decolorized with different concentrations of ethanol (once with $80 \%$ ethanol, 
once with $95 \%$ ethanol and twice with anhydrous ethanol) at room temperature for 2-5 min. Finally, xylene permeated the slices and sealed them with neutral gum at room temperature.

Immunofluorescence double staining. Rabbit anti-human GPR4 polyclonal antibody (Abcam), mouse anti-human CD105 monoclonal antibody (Abcam), goat anti-rabbit Alexa Fluor 488 IgG (Jackson ImmunoResearch; https://www.jacksonimmuno.com/\#) and goat anti-mouse Cy3 IgG (Jackson) were used in immunohistochemistry assays. Tissue sections $(10 \mu \mathrm{m}$ tissue sections embedded with SAKURA Tissue-Tek O.C.T. Compound) were fixed in ice cold acetone $\left(-20^{\circ} \mathrm{C}\right)$ for $10 \mathrm{~min}$ and permeabilized with PBS-Tween-20 (PBST; 0.1\%) at room temperature. Non-specific binding sites were blocked with blocking buffer (10\% goat serum; Zsbio Commerce Store) for $1 \mathrm{~h}$ at room temperature. Incubation was performed with a rabbit anti-human GPR4 polyclonal antibody (catalog no. ab188606; 1:100) and a mouse anti-human CD105 monoclonal antibody (catalog no. ab14114; 1:100), stored overnight at $4^{\circ} \mathrm{C}(>16 \mathrm{~h})$, followed by four washes with PBST and incubated with goat anti-rabbit Alexa Fluor 488 IgG (ab2338840; 1:100) and goat anti-mouse Cy3 IgG (ab2338000; 1:100). The secondary antibody was placed in an opaque wet box for $1 \mathrm{~h}$ at room temperature for incubation. Sections were observed by laser confocal microscopy. PBS was used as a negative control instead of the primary antibody. Immunofluorescence staining of GPR4 and CD105 expression was detected by confocal laser scanning microscopy using 488- and $561 \mathrm{~nm}$ wavelengths to excite green and red fluorescence, respectively.

Laser scanning confocal microscopy. Immunofluorescence staining of GPR4 and CD105 expression was detected by confocal laser scanning microscopy (TCS-SP5; Leica Microsystems $\mathrm{GmbH}$ ). Wavelengths of 488 and $561 \mathrm{~nm}$ were used to excite the green and red fluorescence, respectively. The scanning resolution was $1024 \times 1024$ pixels. At $\times 200$ magnification, three images were randomly captured for each slice. New microvascular ECs (CD105-labeled) appeared green, GPR4-labeled cells appeared red, and CD105- and GPR4-labeled cells appeared yellow.

MVD determination. CD105-positivity was defined by capillaries appearing green upon CD105 staining. These were counted based on the Weidner's standard $(28,29)$. MVD was observed in fields of x100 magnification in the most vascularized areas, termed hotspots. The average vessel count in the three fields at x200 magnification was used as the final MVD value. ECs or EC clusters stained green were counted as a separate blood vessel. A clear separation was required between these vessels. Lumen formation and the presence of red blood cells in the cavity were not included.

GPR4 staining observation. GPR4 expression level was detected by ImageJ analysis software (verison 2.0; National Institutes of Health). The software was used to analyze each images of sections captured at x200 magnification in the three fields. Parameter settings included a selection of parameters commonly used in the literature: Positive area (area), average optical density (mean density) and integrated optical density
(IOD). Image analysis results consisted of the average grayscale value: IOD/area (30).

Analysis of co-expression and co-localization of GPR4 and CD105. Image pro-plus software (version 6.0; Media Cybernetics) was used to analyze confocal images, with correlation coefficients and overlap coefficients. Pearson's correlation coefficients were assigned to colocalization degrees used to measure the linear relationship between distance variables as follows: 0.6-0.8, Strong correlation; 0.4-0.6, medium correlation; 0.2-0.4 weak correlation; and 0.0-0.2 extremely weak correlation or no correlation.

Controls. Non-HCC tissues were collected as the control group. According to the protocol from the antibodies obtained from Abcam, human lung tissues were used as the GPR4 positive control, mice brain tissues as the CD105 positive control and PBS as the negative control. The tissues were derived from surgical excision of lung tissue from a patient that provided written informed consent prior to the study, and from laboratory mice. The use of the mice was approved by the Ethics Committee of the First Affiliated Hospital of Xi'an Jiaotong University (Xi'an, China).

Statistical analysis. The statistical analysis was processed with the statistical software programs Prism (version 7.0; GraphPad) and SPSS (version 18.0; IBM Corp.). Measurement data are expressed as the mean \pm standard deviation. Statistical comparisons of data between two groups were performed with the paired or unpaired Student's t-test; comparisons between different parts of the same specimen were performed with the paired Student's t-test. ANOVA was used to compare multiple mean values. LSD, S-N-K and Tamhane's T2 test was used for the post hoc test following ANOVA. Under other conditions, comparisons were carried out by non-parametric analysis. Pearson's correlation coefficients were used to assess the linear correlation between distant variables. Univariate log-rank test and Kaplan-Meier survival curve were used for the survival analysis.

\section{Results}

Expression of CD105 in HCC and control tissues. Human $\mathrm{HCC}$ tumors and normal liver tissues were compared following hematoxylin staining (Fig. 1A). The tissues were stained for CD105 and GPR4 by immunofluorescence double staining (Fig. 1B). A low level of CD105 was expressed in the control tissues, and was mainly expressed on the cell membrane in HCC tumor tissues. Green fluorescent staining was strongly scattered in tumor tissues and in the ECs of liver tissue within $2 \mathrm{~cm}$ of the tumor. These positively stained single cell or cell clusters mainly formed four types of shapes: Sinus, branching, oval and sprouting. Most of these positively stained ECs formed thin and irregular walls without smooth muscle tissue. The mean MVD, determined using CD105, of the 37 liver tumor tissues was significantly higher than that of the 10 control tissues $(\mathrm{P}<0.05$; mean, 61.51 \pm 28.09 and 12.90 \pm 11.72 , respectively; Table I).

Similar to CD105, GPR4 was mainly expressed on the cell membrane and exhibited a lumen-like structure. The expression of GPR4 was significantly lower in the control 
Table I. Expression of CD105 in HCC and control tissues.

\begin{tabular}{lcccr}
\hline Pathological group & Cases, $\mathrm{n}$ & MVD $^{\mathrm{a}}$ & P-value & $\mathrm{t}$ \\
\hline HCC tissues & 37 & $61.51 \pm 28.09$ & $<0.05$ & 8.210 \\
Control tissues & 10 & $12.90 \pm 11.72$ & & \\
\hline
\end{tabular}

${ }^{a}$ Mean \pm standard deviation. MVD, microvessel density; HCC, hepatocellular carcinoma.
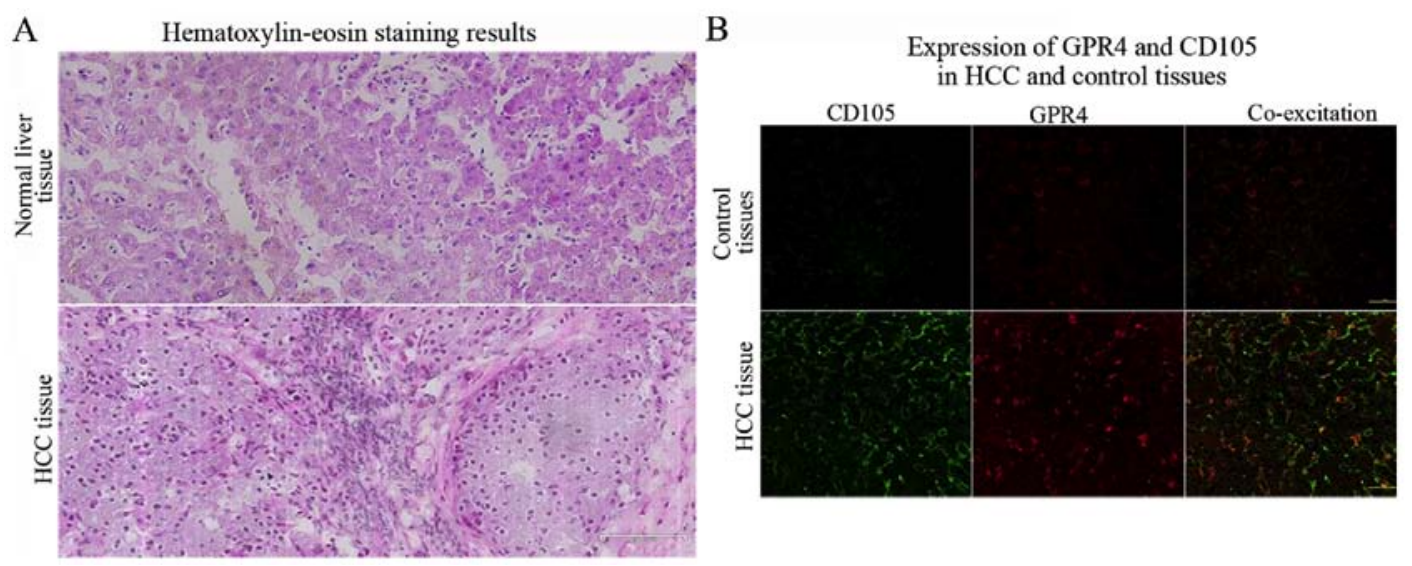

Figure 1. Hematoxylin-eosin staining and expression of GPR4 and CD105 in HCC and control tissues. (A) Hematoxylin-eosin staining of normal liver tissue and HCC tumor tissue. Normal liver cells are radially arranged like a beam, and hepatic sinusoids are visible among the polygonal hepatic cords of the cells. Liver cancer cells appear polygonal and nested, with enlarged nuclei, and interstitial fibrosis, visible hyperplasia of small blood vessels and a small proportion of inflammatory cell infiltration is present. (B) Expression of GPR4 and CD105 in HCC tumor and control tissues. Immunofluorescence double staining of GPR4 (red), CD105 (green) and co-localization of both (yellow) was observed under a confocal laser scanning microscope. CD105 expression was mainly on the cell membrane in HCC tissues and was very low in control tissues. Positive expression of GPR4 was mainly observed in the cell membrane, showing a lumen-like structure in HCC tissue and was weakly expressed in control tissues. Magnification x200. GPR4, G-protein coupled receptor 4; HCC, hepatocellular carcinoma.

tissues compared with that in the HCC tissues $(\mathrm{P}<0.05$; mean, $0.0259 \pm 0.0041$ and $0.0386 \pm 0.0125$, respectively; Table II).

Correlation between GPR4 and CD105 in HCC and control tissues. In control tissues, laser confocal imaging revealed very weak and low co-localized expression of CD105 and GPR4. Pearson's correlation analysis revealed no significant correlation between CD105 and GPR4 co-localization ( $>0.01$; $r=0.28$; Fig. 2C) in control tissues.

Laser confocal imaging revealed that the region with high GPR4 expression was consistent with high expression of CD105, particularly in the sinusoidal and oval ECs of liver tissues $<2 \mathrm{~cm}$ adjacent to the tumor. Co-localization of GPR4 and CD105 was detected as yellow fluorescence. Pearson's correlation analysis revealed a positive correlation between the expression of CD105 and GPR4 ( $<<0.01 ; \mathrm{r}=0.666$; Fig. 2A). The Image-pro-plus software was used to analyze three representative confocal images (Fig. 2B). The Pearson's correlation coefficients indicated strong correlations calculated by the Image-pro-plus software ( $\mathrm{r}=0.65, \mathrm{r}=0.88$ and $\mathrm{r}=0.62)$. These results corroborated the previous observation, further illustrating the association between GPR4 and microvessel formation.

Expression of CD105 in the marginal and central areas of HCC tissues. The positively stained regions for CD105 were found to be scattered in the cancer tissues, particularly in the margin of the invasion extension. However, a lower expression of CD105 was found in the central areas of the neoplastic tissues or in areas of necrosis (Fig. 3). The expression of CD105 in the tumor edge was significantly higher than that in the central area $(\mathrm{P}<0.05$; Table III). This observation could be explained by the higher number of new blood vessels in the peripheral regions of HCC tissues, with intensive neovascularization.

Expression of GPR4 in the marginal and central areas of HCC tissues. The expression of GPR4 in HCC tissues was not significantly different between the marginal and central areas $(\mathrm{P}>0.05$; Fig. 3; Table IV). This may be due to the hypoxic environment in the center of the tumor leading to high GPR4 expression.

Association between CD105 and clinicopathological features. MVD, determined by staining for CD105, was compared between different clinicopathological factors of patients (Table V). This demonstrated that CD105 expression was independent of sex, age, tumor differentiation degree, cirrhosis and ascites $(\mathrm{P}>0.05)$. Conversely, $\mathrm{CD} 105$ was associated with lymphatic invasion (Fig. 4A), tumor size (Fig. 4B), intravascular tumor thrombus (Fig. 4C) and TNM staging (all $\mathrm{P}<0.05)$

Association between GPR4 and clinicopathological features. GPR4 expression was significantly associated with tumor size (Fig. 4B) and blood vessel invasion (Fig. 4C) 
Table II. Expression of GPR4 in HCC and control tissues.

\begin{tabular}{lccr}
\hline Pathological group & Cases, $\mathrm{n}$ & Fluorescence intensity $^{\mathrm{a}}$ & $\mathrm{P}$-value \\
\hline HCC tissues & 37 & $0.0386 \pm 0.0125$ & $<0.05$ \\
Control tissues & 10 & $0.0259 \pm 0.0041$ & 5.210 \\
\hline
\end{tabular}

${ }^{\mathrm{a}}$ Mean \pm standard deviation. HCC, hepatocellular carcinoma.

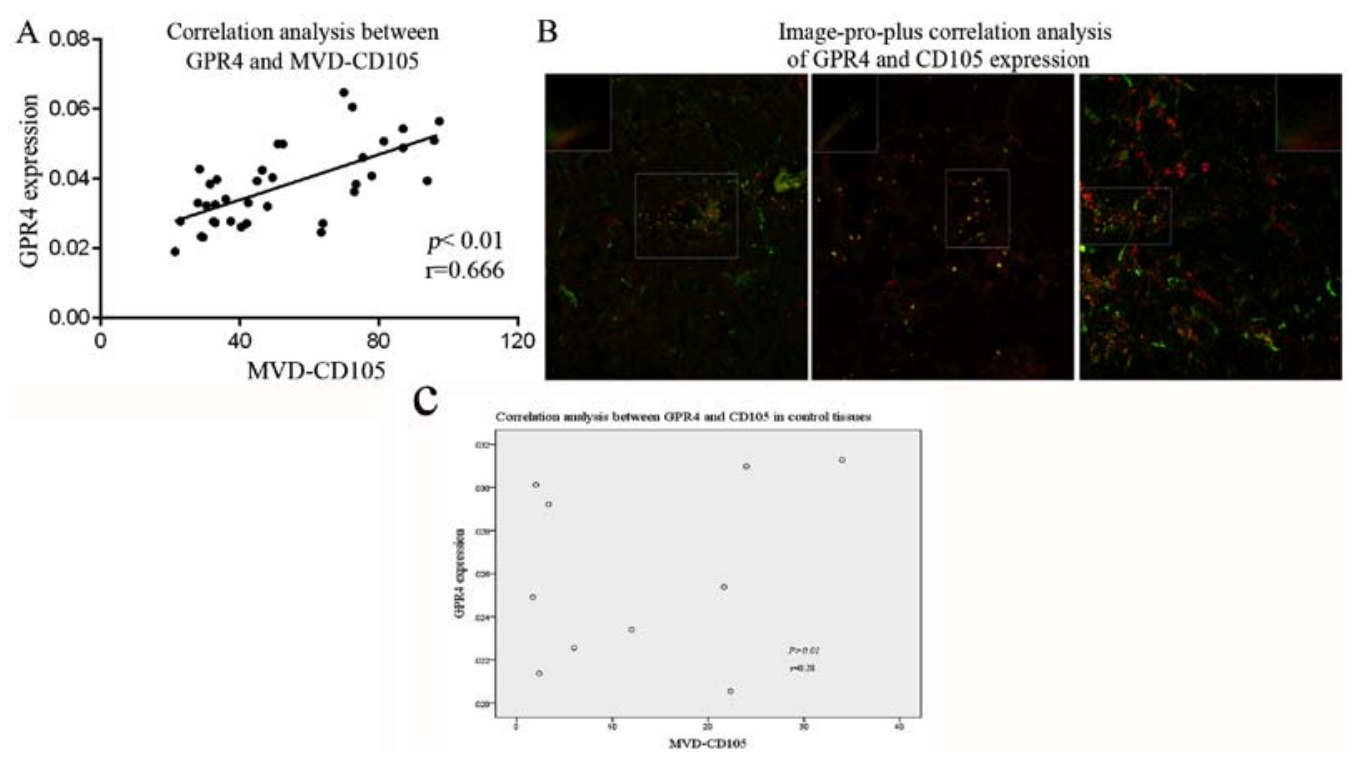

Figure 2. Association between GPR4 expression and MVD-CD105. (A) Scatter plot of GPR4 expression versus MVD-CD105 for correlation analysis in HCC tumor tissues. This is a linear graph of the correlation. (B) Image-pro-plus correlation analysis of GPR4 (red) and CD105 (green) co-localization (yellow). Pearson's correlation coefficients determined by software analysis are as follows: $1, \mathrm{r}=0.65 ; 2, \mathrm{r}=0.88 ; 3, \mathrm{r}=0.62$. Magnification $\mathrm{x} 200$. (C) Scatter plot of GPR4 expression versus MVD-CD105 for correlation analysis in control tissues (Pearson's correlation coefficients: $r=0.28, P>0.01$ ). GPR4, G-protein coupled receptor 4; MVD, microvessel density.

(both $\mathrm{P}<0.05$; Table VI). This may be explained by the high nutrient and oxygen requirements to maintain the biological activity associated with larger tumors and blood vessel invasion, which leads to induction of angiogenesis and GPR4 expression.

Association between GPR4 and CD105 expression and the prognosis of patients with HCC. Postoperative clinical data were retrieved from outpatient medical records or telephone calls. Overall, $91.89 \%$ of patients successfully completed follow-up, with a median follow-up time of 14.5 months (range, 2-36 months). The remaining three patients were lost to follow up. OS time was calculated as the period from surgery until death.

Prognostic significance was investigated by using the average GPR4 expression (mean, 0.0386) and MVD-CD105 (mean, 61.51) levels as the cut-offs to divide patients into low- and high-CD105/GPR4 groups (Fig. 5). OS time was significantly shorter in the high-CD105/GPR4 group compared with that in the low-CD105/GPR4 group.

Survival analysis with the univariate log-rank test showed that MVD-CD105 and GPR4 expression affect the prognosis of patients with HCC, and this was statistically significant $(\mathrm{P}<0.05)$. Kaplan-Meier survival curves suggested that increased MVD-CD105 and GPR4 expression were associated with a higher risk of death in HCC.

\section{Discussion}

In the present study, MVD (based on CD105-labeled ECs) and GPR4 expression were significantly higher in HCC than in control tissues. This suggests the involvement of CD105 and GPR4 in the neovascularization of HCC, and the GPR4 role of $\mathrm{HCC}$ in angiogenesis. Previous studies have shown that traditional vascular markers, such as CD31, are expressed in both small and large blood vessels, and that CD105 is weakly expressed in blood vessels of non-cancerous tissues (24). CD105 is highly expressed in blood vessel ECs both within and surrounding the tumor $(23,24)$. CD105 is mainly expressed during neovascularization, particularly in the ECs of immature tumor vasculature, and is believed to inhibit tumor angiogenesis. This makes it an ideal therapeutic target in cancer $(24,31,32)$. Certain previous studies compared CD105-labeled MVD with CD31-labeled tumor MVD and concluded that CD105-labeled MVD was a risk factor for tumor progression compared with CD31 [P=0.020; hazard ratio, 1.873; 95\% confidence interval, 1.102-3.184) (33). Moreover, CD105-labeled MVD in tumor tissue was associated with tumor progression and prognosis, as 
Table III. Expression of CD105 in HCC.

\begin{tabular}{lcccr}
\hline Region & Cases, $\mathrm{n}$ & MVD $^{\mathrm{a}}$ & P-value & $\mathrm{t}$ \\
\hline Central part of HCC tissues & 37 & $44.49 \pm 19.95$ & $<0.05$ & 6.863 \\
Marginal part of HCC tissues & 37 & $61.51 \pm 28.09$ & & \\
\hline
\end{tabular}

${ }^{\mathrm{a}}$ Mean \pm standard deviation. MVD, microvessel density; HCC, hepatocellular carcinoma.

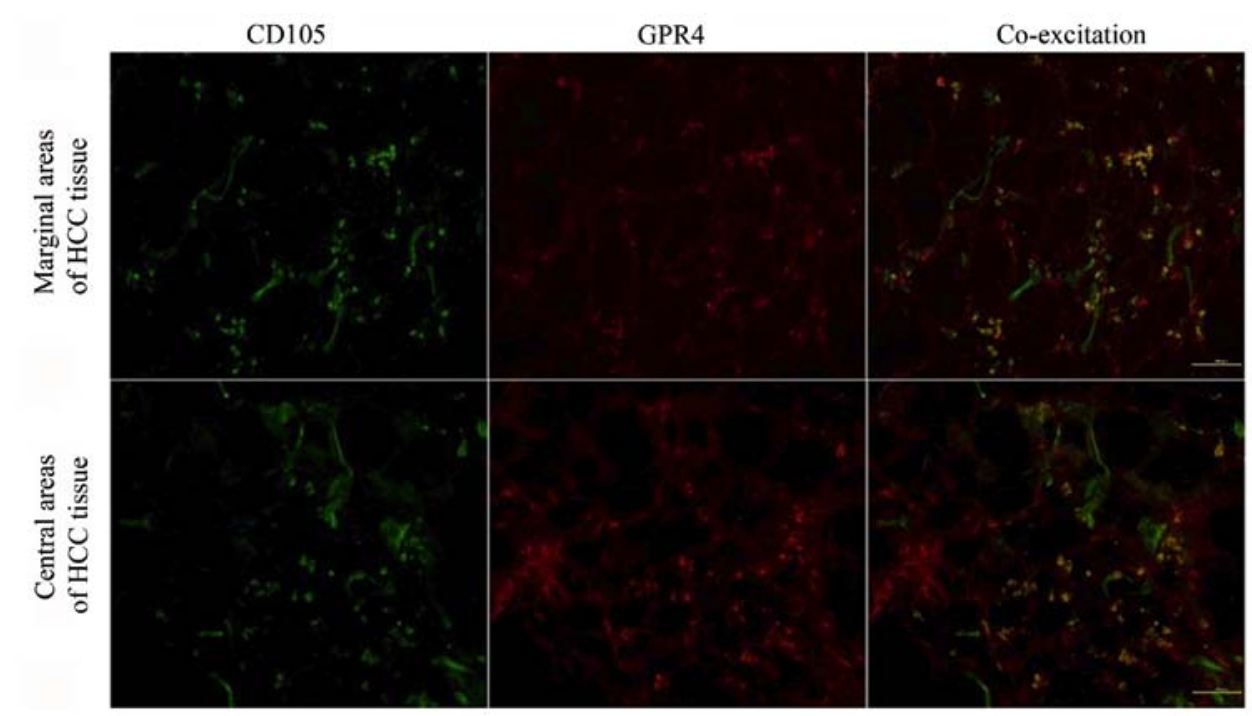

Figure 3. Expression of GPR4 and CD105 in marginal and central regions of HCC tumor tissues. Immunofluorescence double staining of GPR4 (red) and CD105 (green) was observed under laser confocal microscopy in marginal areas and central areas of HCC tissue. Magnification x200. GPR4, G-protein coupled receptor 4; MVD, microvessel density; HCC, hepatocellular carcinoma.

established in several solid tumor studies (32-37), such as prostate cancer, primary hepatocellular carcinomas, non-small cell lung cancer, rectal cancer and oral squamous cell carcinoma. Therefore, CD105 may be more representative and sensitive as a tumor vascular marker compared with a pan-vascular marker in predicting tumor prognosis. Therefore, the expression and clinical significance of CD105 in microvessels may be more sensitive than that of CD31 or CD34. Honda et al (14) reported that neovascularization density was increased in liver cancer, which was consistent the result from previous studies $(23,24,31,32)$.

A significant and positive correlation was demonstrated between GPR4 expression level and MVD in the present study. The localization of high GPR4 expression was consistent with the CD105 expression hotspot in tissues of hepatic sinusoidal and oval ECs $<2 \mathrm{~cm}$ adjacent to the tumor. GPR4 was highly expressed in liver tumor tissues, not only in marginal region. Moreover, the expression of GPR4 was co-localized with CD105, which demonstrates the presence of neovascularization-associated ECs. Therefore, the high expression of GPR4 in the endothelium of neovascularization may be involved in tumor angiogenensis. A review of the relevant literature found no studies on the expression of GPR4 in liver tumor tissues and blood vessels. In the present study, the expression of GPR4 in liver tumor tissues and blood vessels was preliminarily detected. Initially, immunofluorescence histochemical study was conducted on frozen sections. IHC and co-staining with classical markers for tumor angiogenesis was conducted for a detailed morphological study on the paraffin sections of tumors. GPR4 expression was observed in the microvessels of the tumors, indicating its involvement in the microvessel angiogenesis in HCC. Furthermore, the findings also indicated the potential of GPR4 and CD105 as therapeutic targets, and as effective and sensitive markers of tumors in HCC. Certain studies have demonstrated the role of GPR4 in the process of angiogenesis. Jing et al (9) revealed the promotion of angiogenesis by GPR4 in head and neck cancer, and Ren et al (10) reported that the overexpression of GPR4 may be required for angiogenesis in epithelial ovarian carcinomas. The expression of VEGFR2 and the angiogenic response to VEGF was found to be decreased in GPR4-deficient mice (11). Certain studies reported a high perinatal mortality rate and a spontaneous bleeding tendency in embryos and neonates that lacked GPR4 expression (12). Additionally, it was observed that a proportion of subcutaneous blood vessels that were covered with defective vascular smooth muscle cells became dilated and tortuous, resulting in impaired vascular stability and abnormal bleeding (12).

Angiogenesis may be associated with the proton-sensor function of GPR4. The angiogenesis effect is stimulated, and proton concentration is increased in hypoxic or ischemic tissues (38-41). Subsequently, GPR4 senses a change in $\mathrm{pH}$ and induces angiogenesis; the histidine on the GPR4 protein can sense the change in the extracellular $\mathrm{pH}$. At acidic $\mathrm{pH}$ levels, 
Table IV. Expression of GPR4 in HCC.

\begin{tabular}{lccr}
\hline Region & Cases, $\mathrm{n}$ & Fluorescence intensity $^{\mathrm{a}}$ & $\mathrm{P}$-value \\
\hline Central part of HCC tissues & 37 & $0.0375 \pm 0.0114$ & 0.350 \\
Marginal part of HCC tissues & 37 & $0.0386 \pm 0.0125$ & -0.947 \\
\hline
\end{tabular}

${ }^{a}$ Mean \pm standard deviation. HCC, hepatocellular carcinoma.

Table V. Association between expression of CD105 in hepatocellular carcinoma tumor tissues and clinicopathological features.

\begin{tabular}{|c|c|c|c|c|}
\hline Clinicopathological features & Cases, $\mathrm{n}$ & $\mathrm{MVD}^{\mathrm{a}}$ & P-value & $\mathrm{t} / \mathrm{F}$ \\
\hline Age, years & & & 0.110 & 1.641 \\
\hline$<60$ & 23 & $67.00 \pm 29.63$ & & \\
\hline$\geq 60$ & 14 & $52.50 \pm 23.62$ & & \\
\hline Sex & & & 0.604 & -0.532 \\
\hline Male & 30 & $60.53 \pm 29.65$ & & \\
\hline Female & 7 & $65.71 \pm 21.41$ & & \\
\hline Tumor size, $\mathrm{cm}$ & & & $<0.01$ & 3.975 \\
\hline$<5$ & 19 & $46.47 \pm 17.34$ & & \\
\hline$\geq 5$ & 18 & $77.39 \pm 28.85$ & & \\
\hline Differentiated degree & & & 0.857 & 0.155 \\
\hline High & 8 & $56.75 \pm 22.96$ & & \\
\hline Middle & 24 & $63.25 \pm 31.20$ & & \\
\hline Poor & 5 & $60.80 \pm 22.742$ & & \\
\hline Lymph node metastasis & & & $<0.01$ & -6.861 \\
\hline No & 35 & $59.69 \pm 27.78$ & & \\
\hline Yes & 2 & $93.50 \pm 2.12$ & & \\
\hline Ascites & & & 0.197 & -1.337 \\
\hline No & 26 & $57.58 \pm 27.97$ & & \\
\hline Yes & 11 & $70.82 \pm 27.36$ & & \\
\hline Cirrhosis of the liver & & & 0.517 & 0.671 \\
\hline No & 8 & $67.88 \pm 30.97$ & & \\
\hline Yes & 29 & $59.76 \pm 27.56$ & & \\
\hline Intravascular tumor thrombus & & & $<0.01$ & -4.530 \\
\hline No & 28 & $52.50 \pm 24.14$ & & \\
\hline Yes & 9 & $89.56 \pm 20.37$ & & \\
\hline TNM staging & & & 0.003 & -3.258 \\
\hline I-II & 21 & $49.62 \pm 22.70$ & & \\
\hline III-IV & 16 & $77.13 \pm 27.35$ & & \\
\hline
\end{tabular}

${ }^{\mathrm{a}}$ Mean \pm standard deviation. MVD, microvessel density; TNM, Tumor-Node-Metastasis.

activated GPR4 can stimulate the adenylate cyclase to produce a large amount of cyclic AMP, with cAMP subsequently activating the downstream pathway of protein kinase $A$ and cyclic adenylate response element binding to the protein. Ultimately, this process activates the transcription of certain genes, including those coding for angiogenic factors such as VEGF, among others $(42,43)$. Therefore, the role of GPR4 in angiogenesis may be due to the function of its $\mathrm{H}^{+}$receptors. In an extracorporeal aortic ring experiment, microvessel products of GPR4-deficient adult mice were less dependent on the $\mathrm{pH}$ of the culture medium compared with wild-type mice (44). Moreover, the ischemic and hypoxic conditions of the tumor microenvironment activates GPR4 and thereby promotes angiogenesis. Another study with ECs demonstrated that GPR4 promotes angiogenesis via the regulation of $\mathrm{C} / \mathrm{EBP}$ homologous protein (CHOP) in an acidic environment (45). Although these studies have shown that GPR4 promotes angiogenesis, the role of GPR4 in liver cancer was not investigated. 

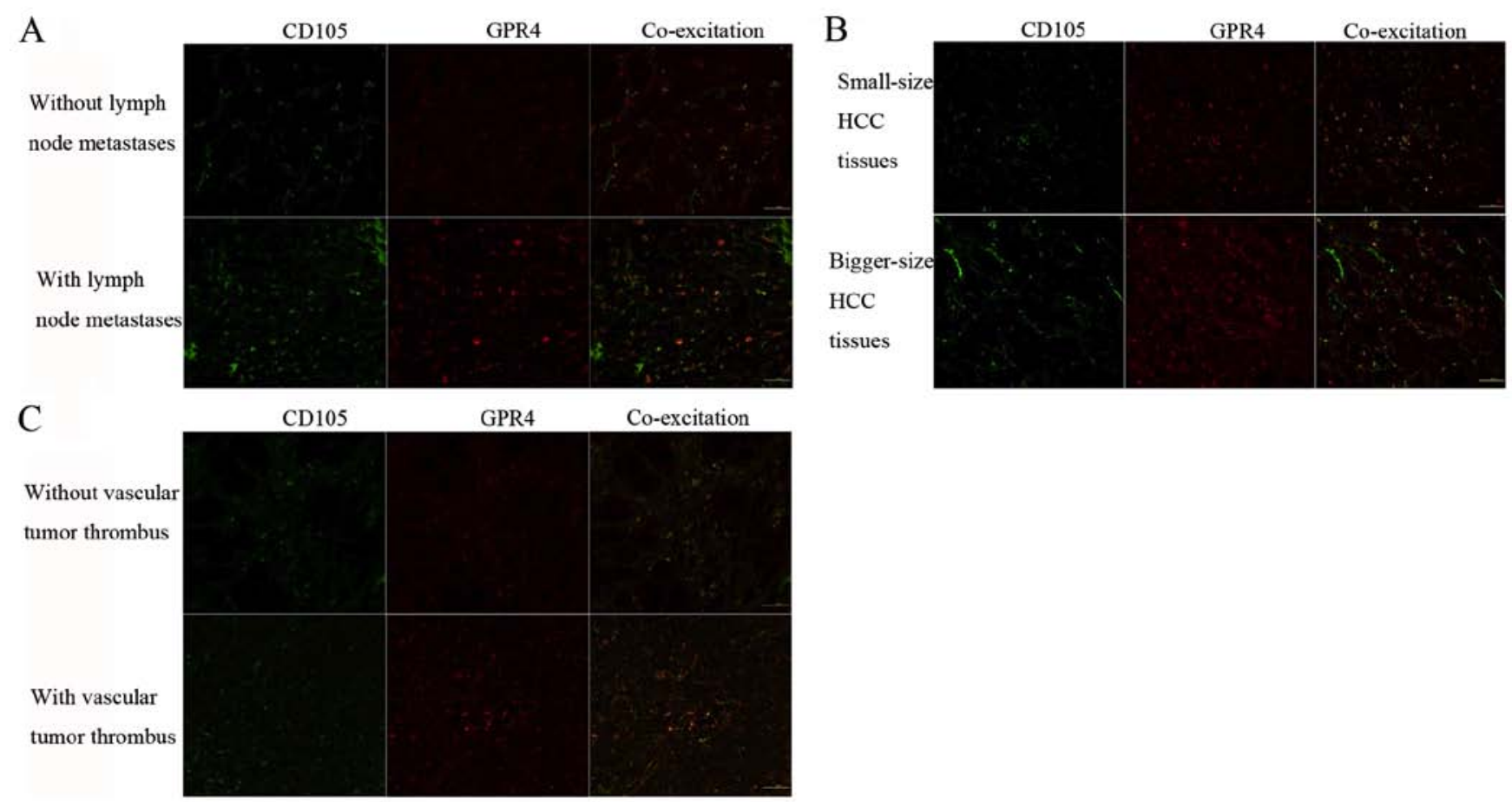

Figure 4. Immunofluorescence double staining of GPR4 and CD105. Immunofluorescence double staining of GPR4 (red) and CD105 (green) was observed under confocal laser scanning microscopy in (A) HCC with versus without lymph node metastases, in (B) HCC of small versus large tumor size and in (C) tumors with versus without vascular tumor thrombus. Magnification x200. GPR4, G-protein coupled receptor 4; MVD, microvessel density; HCC, hepatocellular carcinoma.

In the present study, GPR4 expression was observed in liver tumor tissues. Previous studies have also shown the expression of GPR4 in a number of tumor tissues, including high expression in HCC $(11,13)$, suggesting its association with tumorigenesis. The study by Sin et al (13) indicated that GPR4 is an oncogene and that it was overexpressed in human cancer tissues, including those of the breast, ovary, colon, liver and kidney. It was also shown that the ectopic overexpression of GPR4 led to oncogenic transformation of NIH3T3 fibroblasts. Although the effect of $\mathrm{pH}$ was not assessed in the study, GPR4 was shown to have tumor-promoting activity (13). Furthermore, high expression of GPR4 in hepatoma cells indicated its role in the development of HCC in the present study. In the present study, GPR4 expression was also found to be associated with the clinicopathological features of HCC patients, indicating the involvement of GPR4 in angiogenesis, tumor growth and the development of HCC. Dong et al (46) observed that the inhibition of cell apoptosis led to decreased expression of CHOP by GPR4, following hypoxia/reoxygenation treatment in human umbilical vein ECs. Moreover, the inhibition of GPR4 resulted in decreased renal injury following ischemia-reperfusion and inhibition of cell apoptosis through the suppression of CHOP expression (46) Therefore, GPR4 has potential as a diagnostic marker for the diagnosis of liver cancer, and as a therapeutic target in HCC. However, further studies are required.

Another finding from the present study was that CD105 expression was closely associated with tumor size, lymph node metastasis, intravascular tumor thrombus and TNM staging in the patients with $\mathrm{HCC}$, highlighting its potential as a marker of vascular EC proliferation. It is highly relevant to determine novel targets of antiangiogenesis and CD105 may be a prognostic factor for patients with HCC. The association between increased neovascularization and clinical characteristics in patients with HCC indicated the highly vascularized nature of this malignancy and the part played by angiogenesis. Thus, uncovering the mechanisms underlying angiogenesis in HCC may provide effective and sensitive markers for HCC diagnosis and a specific target for antiangiogenic therapy.

In the present study, differences in mean CD105 values were found between the tumor edge and central area. However, no such difference was observed with GPR4 expression. Furthermore, a higher number of new blood vessels was observed in the peripheral region of HCC tissues, where the proliferation of ECs was more active. Intensive neovascularization elicits hypoxic conditions, inducing the expression of CD105 predominantly in the tumor marginal edge (47). On the other hand, no significant differences in GPR4 expression were observed between the tumor edge and the tumor center in the present study. It is acknowledged that an extracellular acidic environment induces the expression of GPR4 (12). The hypoxic environment in the central tumor area may lead to high GPR4 expression, leading to an absence of differences in GPR4 expression between the neovascular-rich edge compared with the central area of the tumor. GPR4 has dual effects of tumor promotion and inhibition, depending on the cell type and biological background. In the present study, GPR4 expression was affected by different clinicopathological factors, although the potential impact of sample differences should also be considered.

Analysis of survival curves showed shorter survival times in patients with liver cancer exhibiting high GPR4 and CD105 expression levels. Notably, the expression levels of CD105 and GPR4 were consistent with the prognostic trend of these 
Table VI. Association between expression of GPR4 in hepatocellular carcinoma tumor tissues and clinicopathological features.

\begin{tabular}{|c|c|c|c|c|}
\hline Clinicopathological features & Cases, $\mathrm{n}$ & Fluorescence intensity ${ }^{a}$ & P-value & $\mathrm{t} / \mathrm{F}$ \\
\hline Age, years & & & 0.184 & 1.357 \\
\hline$<60$ & 23 & $0.0406 \pm 0.0137$ & & \\
\hline$\geq 60$ & 14 & $0.0353 \pm 0.0099$ & & \\
\hline Sex & & & 0.051 & 2.073 \\
\hline Male & 30 & $0.0399 \pm 0.0133$ & & \\
\hline Female & 7 & $0.0330 \pm 0.0062$ & & \\
\hline Tumor size, $\mathrm{cm}$ & & & $<0.01$ & 4.349 \\
\hline$<5$ & 19 & $0.0314 \pm 0.0064$ & & \\
\hline$\geq 5$ & 18 & $0.0462 \pm 0.01302$ & & \\
\hline Differentiated degree & & & 0.520 & 0.667 \\
\hline High & 8 & $0.0428 \pm 0.0039$ & & \\
\hline Middle & 24 & $0.0370 \pm 0.0027$ & & \\
\hline Poor & 5 & $0.0398 \pm 0.0049$ & & \\
\hline Lymph node metastasis & & & 0.223 & -1.850 \\
\hline No & 35 & $0.0382 \pm 0.0127$ & & \\
\hline Yes & 2 & $0.0462 \pm 0.0053$ & & \\
\hline Ascites & & & 0.286 & -1.103 \\
\hline No & 26 & $0.0371 \pm 0.0119$ & & \\
\hline Yes & 11 & $0.0423 \pm 0.0138$ & & \\
\hline Cirrhosis of the liver & & & 0.596 & -0.543 \\
\hline No & 8 & $0.0367 \pm 0.0105$ & & \\
\hline Yes & 29 & $0.0392 \pm 0.0131$ & & \\
\hline Intravascular tumor thrombus & & & $<0.01$ & -4.457 \\
\hline No & 28 & $0.0349 \pm 0.0114$ & & \\
\hline Yes & 9 & $0.0502 \pm 0.008$ & & \\
\hline TNM staging & & & $<0.01$ & -4.820 \\
\hline I-II & 21 & $0.0318 \pm 0.0094$ & & \\
\hline III-IV & 16 & $0.0476 \pm 0.0102$ & & \\
\hline
\end{tabular}

${ }^{\mathrm{a}}$ Mean \pm standard deviation. MVD, microvessel density; TNM, Tumor-Node-Metastasis.

A Overall survival

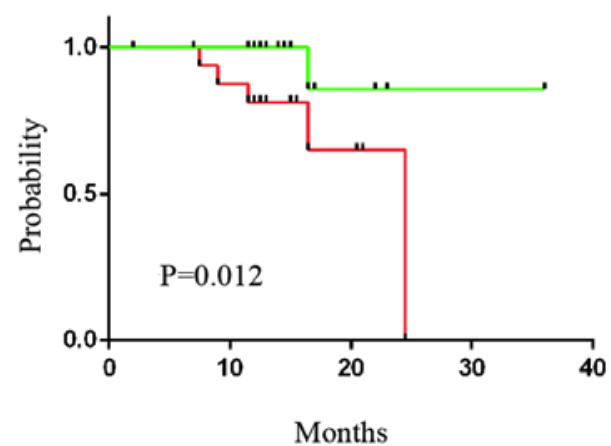

B

Overall survival

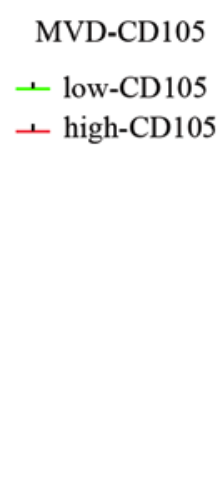

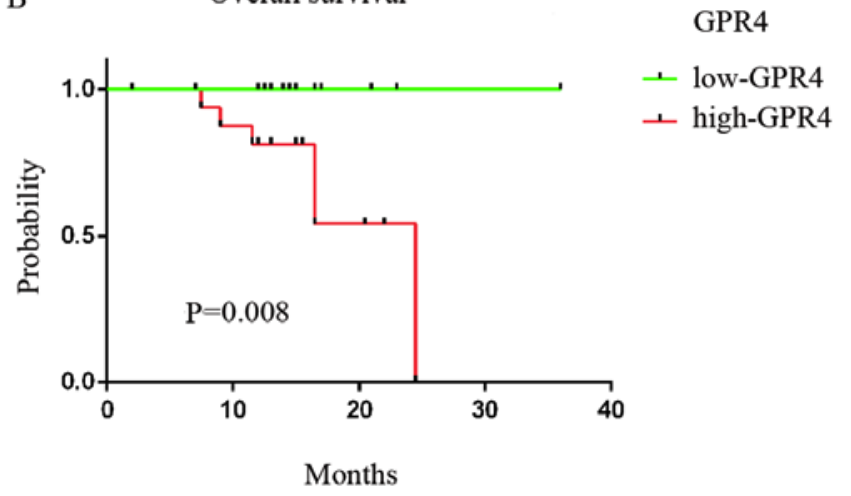

Figure 5. OS times of patients with different CD105/GPR4 expression levels. OS survival time in patients with low or high expression of (A) CD105 (MVD) and (B) GPR4. GPR4, G-protein coupled receptor 4; MVD, microvessel density; OS, overall survival.

patients. This suggests the potential value of using CD105 and GPR4 as predictive markers of prognosis in cancer patients, and this is worth pursuing in further investigations.
Previous studies have implicated GPR4 in two main processes, which were corroborated in the present study. On one hand, GPR4 is involved in angiogenesis in ovarian cancer (10). 
On the other hand, it is an oncogene associated with tumor progression (13). However, it is worth mentioning that the present study was the first to suggest a role for GPR4 in liver cancer, as, to the best of our knowledge, previous studies have not investigated GPR4 in this type of tumor. The present study was the first to investigate the association between GPR4 and MVD in liver tumor tissues, as well as the association between GPR4 and the clinicopathological features of patients with liver cancer.

Due to the limited number of specimens collected, the association between GPR4 and tumor angiogenesis needs to be validated and further studied. Western blot analysis will be carried out in future studies to further assess the expression levels of the proteins investigated in the present study. Furthermore, future studies should focus on the effect of inhibiting and promoting angiogenesis in vitro on GPR4 expression.

Several therapeutic options are available for HCC, including surgical resection, chemotherapy, radiofrequency ablation, transarterial chemoperfusion, selective internal radiation therapy, transarterial chemoembolization, percutaneous ethanol instillation, monoclonal antibody therapy and liver transplantation (48-54). Tumor vascular-targeting therapy has been widely considered in medical research. The rich vascularity of HCC suggests the inhibition of angiogenesis as a therapeutic target in this tumor and indicates that antiangiogenic therapy may be a relevant option for advanced HCC. Angiogenesis in $\mathrm{HCC}$ is regulated by both proangiogenic and antiangiogenic factors. Proangiogenic factors include VEGF, matrix metalloproteases, angiopoietin-2 and basic fibroblast growth factor, and angiogenesis inhibitors include tissue inhibitor of metalloproteinases 1, angiostatin, endostatin and thrombospondin (55-58) Some inflammatory molecules, such as signal transducer and activator of transcription 3 , nuclear factor- $\kappa \mathrm{B}$, tumor necrosis factor- $\alpha$ and interleukin-8, also have potential roles in liver cancer angiogenesis (56-59). Antiangiogenesis therapy of HCC has been gradually diversified, with VEGF inhibitors, tyrosinase receptor inhibitors and drugs that directly inhibit ECs. The targeted drug sorafenib has been widely recognized as a first-line treatment with good clinical efficacy. However, inhibitors of angiogenesis do not completely inhibit or suppress liver cancer angiogenesis (59). The investigation of the various mechanisms of HCC angiogenesis, both at the molecular and genetic levels, is hence highly recommended.

Overall, the results from the present study allow the conclusion that both CD105 and GPR4 are positively expressed in benign and malignant liver tissues. Compared with control tissues, tumor tissues exhibited high CD105 and GPR4 expression. There was a strong positive correlation between the average fluorescence intensities of GPR4 and CD105 (MVD). GPR4 and CD105 were co-localized in the vascular endothelium of cancer tissues. GPR4 was preferentially expressed in the margin compared with the central area of the tumors, suggesting its expression in HCC tumor microvessels and its potential implication in HCC angiogenesis and development. These results indicated the therapeutic relevance of investigating novel antiangiogenic targets. The association between the expression of GPR4 and the clinicopathological features of patients with $\mathrm{HCC}$ further indicated its role in angiogenesis, growth and the prognosis of patients with HCC. The mechanism underlying the role of GPR4 in HCC angiogenesis will be further investigated in future studies.

\section{Acknowledgements}

Not applicable.

\section{Funding}

This manuscript was supported by the National Natural Science Foundations of China (grant no. 81772793/H1621, 31201060/C0709, 30973175/C1701 and 81172490/H1621), the Program for New Century Excellent Talents in University (grant no. NCET-12-0440), the Scientific and Technological Research Foundation of Shaanxi Province (grant no. 2012K13-01-06), the Research Foundation of Health Department of Shaan'xi Province (grant no. 2010D41), the Fundamental Research Funds for the Central Universities, First Affiliated Hospital of Xi'an Jiaotong University, and the Clinical Research Award of the First Affiliated Hospital of Xi'an Jiaotong University (grant no. XJTU1AHCR2014-041).

\section{Availability of data and materials}

The datasets used and/or analysed during the present study are available from the corresponding author on reasonable request.

\section{Authors' contributions}

JR conceived and supervised the study; JR and CFX designed the experiments; JR, CFX, SS, YLY, SY, SHB and YYW performed the experiments; LYC, JZZ, RL, XZZ and HLM analyzed the data; JR, CFX, SS and HLM wrote the manuscript; JR and XZZ revised the manuscript. All authors have read and approved the final version of this submission.

\section{Ethics approval and consent to participate}

This study was approved by the Ethics Committee of Xi'an Jiaotong University. Written informed consent was obtained from each patient, including consent for their samples to be taken and used for research purposes, before surgery.

\section{Patient consent for publication}

Not applicable.

\section{Competing interests}

The authors declare that they have no competing interests.

\section{References}

1. Ludwig MG, Vanek M, Guerini D, Gasser JA, Jones CE, Junker U, Hofstetter H, Wolf RM and Seuwen K: Proton-sensing G-protein-coupled receptors. Nature 425: 93-98, 2003.

2. Liang B, Lei W and Gaole A: Relationship between proton-sensing G-protein coupled receptors and tumorigenesis and tumor metastasis. Chin J Biochem Mol Biol 2011 (In Chinese).

3. Murakami N, Yokomizo T, Okuno T and Shimizu T: G2A is a proton-sensing G-protein-coupled receptor antagonized by lysophosphatidylcholine. J Biol Chem 279: 42484-42491, 2004.

4. Wang JQ, Kon J, Mogi C, Tobo M, Damirin A, Sato K, Komachi M, Malchinkhuu E, Murata N, Kimura T, et al: TDAG8 is a proton-sensing and psychosine-sensitive G-protein-coupled receptor. J Biol Chem 279: 45626-45633, 2004. 
5. Mahadevan MS, Baird S, Bailly JE, Shutler GG, Sabourin LA, Tsilfidis C, Neville CE, Narang M and Korneluk RG: Isolation of a novel G protein-coupled receptor (GPR4) localized to chromosome 19q13.3. Genomics 30: 84-88, 1995

6. Justus CR, Dong L and Yang LV: Acidic tumor microenvironment and $\mathrm{pH}$-sensing $\mathrm{G}$ protein-coupled receptors. Front Physiol 4: 354, 2013.

7. Ren J, Zhang Y, Cai H, Ma H, Zhao D, Zhang X, Li Z, Wang S, Wang J, Liu R, et al: Human GPR4 and the notch signaling pathway in endothelial cell tube formation. Mol Med Rep 14: 1235-1240, 2016.

8. Kim KS, Ren J, Jiang Y, Ebrahem Q, Tipps R, Cristina K Xiao YJ, Qiao J, Taylor KL, Lum H, et al: GPR4 plays a critical role in endothelial cell function and mediates the effects of sphingosylphosphorylcholine. FASEB J 19: 819-821, 2005.

9. Jing Z, Xu H, Chen X, Zhong Q, Huang J, Zhang Y, Guo W, Yang Z, Ding S, Chen $\mathrm{P}$ and Huang $\mathrm{Z}$ : The proton-sensing G-protein coupled receptor GPR4 promotes angiogenesis in head and neck cancer. PLoS One 11: e0152789, 2016.

10. Ren J, Jin W, Gao YE, Zhang Y, Zhang X, Zhao D, Ma H, Li Z, Wang J, Xiao L, et al: Relations between GPR4 expression, microvascular density (MVD) and clinical pathological characteristics of patients with epithelial ovarian carcinoma (EOC). Curr Pharm Des 20: 1904-1916, 2014

11. Wyder L, Suply T, Ricoux B, Billy E, Schnell C, Baumgarten BU, Maira SM, Koelbing C, Ferretti M, Kinzel B, et al: Reduced pathological angiogenesis and tumor growth in mice lacking GPR4, a proton sensing receptor. Angiogenesis 14: 533-544, 2011.

12. Yang LV, Radu CG, Roy M, Lee S, McLaughlin J, Teitell MA, Iruela-Arispe ML and Witte ON: Vascular abnormalities in mice deficient for the $\mathrm{G}$ protein-coupled receptor GPR4 that functions as a pH sensor. Mol Cell Biol 27: 1334-1347, 2007.

13. Sin WC, Zhang Y, Zhong W, Adhikarakunnathu S, Powers S Hoey T, An S and Yang J: G protein-coupled receptors GPR4 and TDAG8 are oncogenic and overexpressed in human cancers. Oncogene 23: 6299-6303, 2004.

14. Honda H, Tajima T, Kajiyama K, Kuroiwa T, Yoshimitsu K, Irie $\mathrm{H}$, Aibe $\mathrm{H}$ Shimada $\mathrm{M}$ and Masuda $\mathrm{K}$ : Vascular changes in hepatocellular carcinoma: Correlation of radiologic and pathologic findings. AJR Am J Roentgenol 173: 1213-1217, 1999.

15. Matsui O, Kobayashi S, Sanada J, Kouda W, Ryu Y, Kozaka K, Kitao A, Nakamura K and Gabata T: Hepatocelluar nodules in liver cirrhosis: Hemodynamic evaluation (angiography-assisted CT) with special reference to multi-step hepatocarcinogenesis Abdom Imaging 36: 264-272, 2011.

16. Hui LI, Qing YE, Yin XJ and Li YM: The research of angiogenesis in hepatocellular carcinoma. Chin Clin Oncol 4, 2009 (In Chinese)

17. Yamamoto K: Clinical management of hepatocellular carcinoma J Okayama Med Association 120: 279-284, 2008.

18. Kin M, Torimura T, Ueno $\mathrm{T}$, Inuzuka $\mathrm{S}$ and Tanikawa $\mathrm{K}$ : Sinusoidal capillarization in small hepatocellular carcinoma. Pathol Int 4: 771-778, 1994.

19. Peng WT, Sun WY, Li XR, Sun JC, Du JJ and Wei W: Emerging roles of $\mathrm{G}$ protein-coupled receptors in hepatocellular carcinoma. Int J Mol Sci 19: 1366, 2018.

20. Chen Y, Teng F, Wang G and Nie Z: Overexpression of CXCR7 induces angiogenic capacity of human hepatocellular carcinoma cells via the AKT signaling pathway. Oncol Rep 36: 2275-2281, 2016.

21. Nassiri F, Cusimano MD, Scheithauer BW, Rotondo F, Fazio A, Yousef GM, Syro LV, Kovacs K and Lloyd RV: Endoglin (CD105): A review of its role in angiogenesis and tumor diagnosis, progression and therapy. Anticancer Res 31: 2283-2290, 2011.

22. Kumar S, Ghellal A, Li C, Byrne G, Haboubi N, Wang JM and Bundred N: Breast carcinoma: Vascular density determined using CD105 antibody correlates with tumor prognosis. Cancer Res 59: 856-861, 1999.

23. Fonsatti E, Altomonte M, Nicotra MR, Natali PG and Maio M: Endoglin (CD105): A powerful therapeutic target on tumor-associated angiogenetic blood vessels. Oncogene 22: 6557-6563, 2003

24. Dallas NA, Samuel S, Xia L, Fan F, Gray MJ, Lim SJ and Ellis LM: Endoglin (CD105): A marker of tumor vasculature and potential target for therapy. Clin Cancer Res 14: 1931-1937, 2008

25. Fonsatti E, Nicolay HJ, Altomonte M, Covre A and Maio M: Targeting cancer vasculature via endoglin/CD105: A novel antibody-based diagnostic and therapeutic strategy in solid tumours. Cardiovasc Res 86: 12-19, 2010
26. Robboy SJ: Surgical Pathology Dissection, an Illustrated Guide. International Journal of Gynecological Pathology. 16: 86, 1997.

27. Edge SB and Compton CC: The American joint committee on cancer: The 7th edition of the AJCC cancer staging manual and the future of TNM. Ann Surg Oncol 17: 1471-1474, 2010.

28. Chen A, Dong L, Leffler NR, Asch AS, Witte ON and Yang LV: Activation of GPR4 by acidosis increases endothelial cell adhesion through the cAMP/Epac pathway. PLoS One 6: e27586, 2011.

29. Le D: Tumor angiogenesis and metastasis-correlation in invasive breast carcinoma. N Engl J Med 324: 1-8, 1991.

30. Yu P, Bu H, Wang H, Zhao G, Zhang J and Zhou Q: Comparative study on image analysis and manual counting of immunohistochemistry. Sheng Wu Yi Xue Gong Cheng Xue Za Zhi 20: 288-290, 2003 (In Chinese).

31. Henry-Berger J, Mouzat K, Baron S, Bernabeu C, Marceau G, Saru JP, Sapin V, Lobaccaro JM and Caira F: Endoglin (CD105) expression is regulated by the liver $\mathrm{X}$ receptor alpha $(\mathrm{NR} 1 \mathrm{H} 3)$ in human trophoblast cell line JAR. Biol Reprod 78: 968-975, 2008.

32. Wikström P, Lissbrant IF, Stattin P, Egevad L and Bergh A: Endoglin (CD105) is expressed on immature blood vessels and is a marker for survival in prostate cancer. Prostate 51: 268-275, 2002.

33. Darcy KM and Birrer MJ: Translational research in the gynecologic oncology group: Evaluation of ovarian cancer markers, profiles, and novel therapies. Gynecol Oncol 117: 429-439, 2010.

34. Yao Y, Pan Y, Chen J, Sun X, Qiu Y and Ding Y: Endoglin (CD105) expression in angiogenesis of primary hepatocellular carcinomas: Analysis using tissue microarrays and comparisons with CD34 and VEGF. Ann Clin Lab Sci 37: 39-48, 2007.

35. Tanaka F, Otake Y, Yanagihara K, Kawano Y, Miyahara R, Li M, Yamada T, Hanaoka N, Inui K and Wada H: Evaluation of angiogenesis in non-small cell lung cancer: Comparison between anti-CD34 antibody and anti-CD105 antibody. Clin Cancer Res 7: 3410-3415, 2001 .

36. Goldiş DS, Sferdian MF, Tarţă C, Fulger LO, Totolici BD and Neamţu C: Comparative analysis of microvessel density quantified through the immunohistochemistry expression of CD34 and CD105 in rectal cancer. Rom J Morphol Embryol 56: 419-424, 2015.

37. Mărgăritescu C, Simionescu C, Mogoantă L, Badea P, Pirici D Stepan A and Ciurea R: Endoglin (CD105) and microvessel density in oral squamous cell carcinoma. Rom J Morphol Embryol 49: 321-326, 2008.

38. Nedergaard M, Kraig RP, Tanabe J and Pulsinelli WA: Dynamics of interstitial and intracellular $\mathrm{pH}$ in evolving brain infarct. Am J Physiol 260: R581-R588, 1991.

39. Lardner A: The effects of extracellular $\mathrm{pH}$ on immune function. J Leukoc Biol 69: 522-530, 2001.

40. Wahl ML and Grant DS: Effects of microenvironmental extracellular $\mathrm{pH}$ and extracellular matrix proteins on angiostatin's activity and on intracellular pH. Gen Pharmacol 35: 277-285, 2000.

41. Gatenby RA and Gillies RJ: Why do cancers have high aerobic glycolysis? Nat Rev Cancer 4: 891-899, 2004.

42. Wang X and Klein RD: Prostaglandin E 2 induces vascular endothelial growth factor secretion in prostate cancer cells through EP2 receptor-mediated cAMP pathway. Mol Carcinog 46: 912-923, 2007.

43. Ristori C, Ferretti ME, Pavan B, Cervellati F, Casini G, Catalani E, Dal Monte M and Biondi C: Adenylyl Cyclase/cAMP System involvement in the antiangiogenic effect of somatostatin in the retina. Results from transgenic mice. Neurochem Res 33: 1247,2008

44. Rozengurt E: Mitogenic signaling pathways induced by $\mathrm{G}$ protein-coupled receptors. J Cell Physiol 213: 589-602, 2007.

45. Dong L, Krewson EA and Yang LV: Acidosis activates endoplasmic reticulum stress pathways through GPR4 in human vascular endothelial cells. Int J Mol Sci 18: 278, 2017.

46. Dong B, Zhang X, Fan Y, Cao S and Zhang X: GPR4 knockout improves renal ischemia-reperfusion injury and inhibits apoptosis via suppressing the expression of CHOP. Biochem J 474: 4065-4075, 2017

47. Li C, Guo B, Wilson PB, Stewart A, Byrne G, Bundred N and Kumar S: Plasma levels of soluble CD105 correlate with metastasis in patients with breast cancer. Int J Cancer 89: 122-126, 2000.

48. Abou-Alfa GK, Amadori D, Santoro A, Figer A, De Greve J, Lathia C, Voliotis D, Anderson S, Moscovici M and Ricci S: Safety and efficacy of sorafenib in patients with hepatocellular carcinoma (HCC) and child-pugh A versus B cirrhosis. Gastrointest Cancer Res 4: 40-44, 2011 
49. Branco F, Bru C, Vilana R, Bianchi L and Alvas de Mattos AA: Percutaneous ethanol injection before liver transplantation in the hepatocellular carcinoma. Ann Hepatol 8: 220-227, 2009.

50. Kawai S, Okamura J, Ogawa M, Ohashi Y, Tani M, Inoue J, Kawarada Y, Kusano M, Kubo Y and Kuroda C: Prospective and randomized clinical trial for the treatment of hepatocellular carcinoma-a comparison of lipiodol-transcatheter arterial embolization with and without adriamycin (first cooperative study). The cooperative study group for liver cancer treatment of Japan. Cancer Chemother Pharmacol 31 (Suppl): S1-S6, 1992.

51. Lau WY, Lai EC and Leung TW: Current role of selective internal irradiation with yttrium-90 microspheres in the management of hepatocellular carcinoma: A systematic review. Int J Rad Oncol Biol Phys 81: 460-467, 2011.

52. Livraghi T, Goldberg SN, Lazzaroni S, Meloni F, Solbiati L and Gazelle GS: Small hepatocellular carcinoma: Treatment with radio-frequency ablation versus ethanol injection. Radiology 210 : 655-661, 1999.

53. Nicolini A, Fasani P, Manini MA, Martinetti L, Forzenigo LV, Iavarone M, Crespi S, Rossi G, Biondetti P, Colombo M and Sangiovanni A: Transarterial embolization with microspheres in the treatment of monofocal HCC. Dig Liver Dis 41: 143-149, 2009.

54. Sun W: Treatment of inoperable HCC after sorafenib: Where will the new paradigm take Us? Gastrointest Cancer Res 2: 49-50, 2008 .
55. Germano G, Allavena P and Mantovani A: Cytokines as a key component of cancer-related inflammation. Cytokine 43: 374-379, 2008

56. Sakurai T, He G, Matsuzawa A, Yu GY, Maeda S, Hardiman G and Karin M: Hepatocyte necrosis induced by oxidative stress and IL- $1 \alpha$ release mediate carcinogen-induced compensatory proliferation and liver tumorigenesis. Cancer Cell 14: 156-165, 2008.

57. Sun HC and Tang ZY: Angiogenesis in hepatocellular carcinoma: The retrospectives and perspectives. J Cancer Res Clin 130: 307-319, 2004.

58. Zhu AX: Systemic treatment of hepatocellular carcinoma: Dawn of a new era? Ann Surg Oncol 17: 1247-1256, 2010.

59. Abdel-Rahman $\mathrm{O}$ and Cheung WY: The expanding role of systemic therapy in the management of hepatocellular carcinoma. Can J Gastroenterol Hepatol 2018: 1-4, 2018.

(i) () () This work is licensed under a Creative Commons

EY NC NO Attribution-NonCommercial-NoDerivatives 4.0 International (CC BY-NC-ND 4.0) License. 